\title{
Cycles in Zero-Sum Differential Games and Biological Diversity
}

TUNG MAI, Georgia Institute of Technology

MILENA MIHAIL, Georgia Institute of Technology

IOANNIS PANAGEAS, MIT

WILL RATCLIFF, Georgia Institute of Technology

VIJAY VAZIRANI, UC Irvine

PETER YUNKER, Georgia Institute of Technology

Negative frequency-dependent selection (i.e., declining fitness with increased frequency in the population) is thought to be one of the factors that maintains biological diversity. In this paper, we give a concrete mathematical argument supporting this. Our model is as follows: A collection of species derive their fitnesses via a rock-paper-scissors-type game whose precise payoffs are a function of the environment. The new aspect of our model lies in adding a feedback loop: the environment changes according to the relative fitnesses of the species (hence, payoffs change as a function of fitness, which in turn changes as a function of payoffs). The changes in the payoffs are in keeping with the principle of negative frequency-dependent selection, which is widespread in nature. In order to model our game as a continuous time dynamical system, we cast it in the setting of a differential game. We show that for certain parameters, this dynamics cycles, i.e., no species goes extinct and diversity is maintained.

We believe that our techniques can be applied to optimization and machine learning to show that first order methods (e.g., gradient descent/ascent) do cycle even in online settings in which the loss function changes with time.

CCS Concepts: • Theory of computation $\rightarrow$ Convergence and learning in games; Convergence and learning in games;

\section{INTRODUCTION}

Game theory has yielded deep insights into biological phenomena for almost a century. For example, the work of Fisher, Haldane, and Wright gave the central model of replicator dynamics which has been used extensively to study evolutionary processes. In this paper, we use game theory to give a concrete mathematical argument supporting the belief that negative frequency-dependent selection maintains biological diversity.

Negative frequency-dependent selection (i.e., declining fitness with increased frequency in the population) is widespread in biology, and is thought to be one of the main factors maintaining biological diversity within a community $[24,30]$. There are many different mechanisms that reduce the fitness of common genotypes or species, but two interactions are nearly ubiquitous, operating

Vijay Vazirani was supported by NSF Grant 1815901.

Authors' addresses: Tung Mai, Georgia Institute of Technology, tmai9@gatech.edu; Milena Mihail, Georgia Institute of Technology, milena.mihail@gmail.com; Ioannis Panageas, MIT, ioannis@csail.mit.edu; Will Ratcliff, Georgia Institute of Technology, will.ratcliff@biology.gatech.edu; Vijay Vazirani, UC Irvine, vazirani@ics.uci.edu; Peter Yunker, Georgia Institute of Technology, peter.yunker@gatech.edu.

Permission to make digital or hard copies of all or part of this work for personal or classroom use is granted without fee provided that copies are not made or distributed for profit or commercial advantage and that copies bear this notice and the full citation on the first page. Copyrights for components of this work owned by others than ACM must be honored. Abstracting with credit is permitted. To copy otherwise, or republish, to post on servers or to redistribute to lists, requires prior specific permission and/or a fee. Request permissions from permissions@acm.org.

(C) 2018 Association for Computing Machinery.

ACM EC'18, fune 18-22, 2018, Ithaca, NY, USA. ACM ISBN 978-1-4503-5829-3/18/06 . \$15.00

https://doi.org/10.1145/3219166.3219227 
at all levels of biological complexity, from microbes to metazoans. First, common organisms tend to be more susceptible to natural enemies, like predators [24], parasites [19] and pathogens [32, 36]. Pathogens themselves also face negative frequency-dependent selection [12] as they co-evolve with a rapidly-changing host population. Natural selection often favors traits that increase diversity, allowing common organisms to produce uncommon offspring. Perhaps the best example of this is the evolution of eukaryotic sex, which may have evolved and persisted by allowing common genotypes to generate rare, pathogen-resistant offspring genotypes [23]. Second, the intensity of competition for limiting resources (e.g., food [10], reproductive opportunities [11], etc.) often scales with frequency. In fact, a classic pattern observed within community ecology is âĂŸniche partitioningâĂŹ [7], in which organisms that compete for different limiting resources stably coexist. When organisms compete for the same limiting resource, the most competitive organism for the shared limiting resource usually ends up dominating the population [37].

When fitness is frequency dependent, game theoretic approaches that use fixed payoff matrices do not accurately represent the behavior of the system. One approach is to make the games dynamic, allowing the payoff matrix to change with time and organismal frequency. Two main categories of such games are stochastic games [34] which are discrete, with the payoff matrix being governed by a Markov chain, and differential games [14], the state space of which is described via a differential equation (continuous time dynamical system). An example of the first is [21], which studies evolution via the so-called 'multiplicative weights update' algorithm, and an example of the second is the recent elegant model of Weitz et al. [38], studying a dynamical version of the tragedy of the commons.

The work presented here is inspired by the last paper, [38]. A tragedy of the commons occurs when a large number of agents simultaneously play a Prisoner's Dilemma-type game. Selfish behavior on the part of agents, i.e., defection, leads to a highly sub-optimal outcome as compared to the globally best outcome in which all agents cooperate. An early, and motivating example, of this phenomena was cattle grazing in a common pasture. Each shepherd's selfish strategy of letting his cattle overgraze leads to a depleted pasture land. The novel idea in [38] was to model a situation in which the actions of the agents slowly change the environment, and hence the payoff matrix, so that the selfish strategy of agents itself changes. They give differential equations modeling this situation and show regimes of parameters under which the game cycles between the two extremes of replete and depleted environments, with selfish behavior cycling between cooperation and defection.

The underlying game analyzed by Weitz et al. is particularly simple in that each agent has only two strategies. In this paper, we consider a more complex class of games which are non-transitive, namely rock-paper-scissors, which has three strategies, and its generalization to $n$ strategies. The rock-paper-scissors (RPS) game belongs to a general class of negative feedbacks in biology caused by non-transitivity. In such scenarios, there is no global optimum, as each strategy beats one of the other two, and is beaten by a third. Non-transitive dynamics appear to be widespread in biology, and rock-paper-scissors games have been reported in diverse organisms, including plants $[4,20]$, animals [35], and microorganisms [16-18]. In general, rock paper scissors dynamics maintain biological diversity (i.e., individuals, genes, or species), as no single strategy is capable of outright dominance [17, 20, 31]. Most prior models of RPS dynamics used in biology assumed fixed payoff matrices. This is an incongruity with biology, where the fitness of most strategies will be frequencydependent. On the other hand, analyzing complex games, such as RPS, in the setting of differential games is not straightforward and there is a need to develop techniques for doing this.

Differential games: In a differential game, payoffs change continuously with time and are defined over a continuous state space. The state variables of such a game evolve over time according 
to a differential equation. These games were first introduced by Isaacs [15] to study pursuerevader games, with military applications in mind. Over the years, such games have had numerous applications in control theory, especially as applied to aerospace, e.g., see [39], and later in economics, e.g., see [6]. To the best of our knowledge, [38] is the first application of such games to biology.

Our model: Our model is as follows: We assume that the fitness of a collection of species is given by a rock-paper-scissors-type game. Additionally, the precise payoff matrix is a function of the environment. The new aspect of our model lies in adding a feedback loop: the environment changes according to the relative fitnesses of the species; in particular, it gives a boost to the species having smaller populations. We cast our model in the setting of a differential game and we show that for a certain setting of parameters, this dynamics cycles.

More precisely, assume a population of $n$ species and let $x$ give the relative population of each species at any point in time, i.e., $\boldsymbol{x}_{i}$ gives the fraction of population of species $i$. We define $\boldsymbol{P}_{1}, \ldots, \boldsymbol{P}_{n}$ to be $n$ RPS-type payoff matrices, where $\boldsymbol{P}_{i}$ favors species $i$ over other species. At any point in time, the payoff matrix $\boldsymbol{P}$ is a convex combination of these $n$ matrices given by weights $\boldsymbol{w}$. We now define two replicator dynamics:

(1) The population $x$ follows a replicator dynamics based on payoff matrix $P$.

(2) The weights $\boldsymbol{w}$ change according to a second replicator dynamics, based on $x$, in such a way that for smaller population species $i$, the weight of $\boldsymbol{P}_{i}$ tends to increase, hence giving species $i$ a boost.

\subsection{Our techniques}

For the model defined above, we show that the dynamics as defined in (1) "cycle" in the following sense: For all but a measure zero of initial population vectors $x_{0}>0$ and weight vectors $\boldsymbol{w}_{0}>0$ (strictly positive coordinates) and for any $\epsilon>0$, the trajectory of (1) will return to a distance at most $\epsilon$ from $\left(\boldsymbol{x}_{0}, \boldsymbol{w}_{0}\right)$ infinitely often (for an illustration of the theorem, see Figures 1,2). In order to prove this recurrence we follow a proof outline that was developed first by in [28] and [22]. The proof relies on the Poincaré recurrence theorem 2.3, a well known theorem established a century ago. In words, the theorem says that systems that satisfy the two conditions of conservation of volume and that no orbit goes to the boundary will, after finite time, return to a state very close to the initial state and this will happen an infinite number of times. We proved that under a certain homeomorphic transformation $\Pi$ (see Definition 3.2), our dynamics satisfies both conditions of Poincaré's theorem.

We established the second condition (Lemma 3.3) by coming up with a log-barrier function (a similar idea is used in constrained optimization) which is finite in the interior of the state space and infinite at the boundary and by proving that this function is constant with respect to time. This suffices to prove that the orbits do not reach the boundary of $\Delta_{n} \times \Delta_{n}$ (and hence the orbits for the transformed system under $\Pi$ are bounded). The former condition can be proved via Liouville's theorem 2.2 (see also [33]). Finally, it can be shown that $\Pi^{-1}$ exists and is continuous, hence the result for the transformed dynamics carries over to the original dynamics (1). Our results hold for any dimensions in contrast to [38]; their results hold for 2 dimensions $(2 \times 2$ payoff matrices $)$ only.

\subsection{Model}

1.2.1 Classic RPS. RPS is a game between two players, each of whom has three strategies. The payoff matrix can be written as

$$
\boldsymbol{P}=\left[\begin{array}{ccc}
0 & -1 & 1 \\
1 & 0 & -1 \\
-1 & 1 & 0
\end{array}\right]
$$


The RPS game can be generalized to more than three strategies, with the $n \times n$ payoff matrix being:

$$
\boldsymbol{P}=\left[\begin{array}{cccccccc}
0 & -\alpha & 0 & 0 & \ldots & 0 & 0 & \alpha \\
\alpha & 0 & -\alpha & 0 & \ldots & 0 & 0 & 0 \\
\vdots & \vdots & \vdots & \vdots & \vdots & \vdots & \vdots & \vdots \\
0 & 0 & 0 & 0 & \ldots & \alpha & 0 & -\alpha \\
-\alpha & 0 & 0 & 0 & 0 & \ldots & \alpha & 0
\end{array}\right] \text {, where } \alpha>0
$$

In the context of biology, we will have $n$ competing species corresponding to the $n$ strategies, Let $x$ be the population vector where $x_{i}$ is the fraction of the population that is species $i$ and $r$ be the fitness vector. Note that $r$ can be computed given $\boldsymbol{x}$, i.e., $\boldsymbol{r}=\boldsymbol{P} \boldsymbol{x}$. The replicator dynamics under $\boldsymbol{P}$ is

$$
x_{i}^{\prime}=x_{i}\left(r_{i}-\bar{r}\right) \quad \forall i,
$$

where $\bar{r}=\boldsymbol{x}^{\top} \boldsymbol{P} \boldsymbol{x}$ is the average fitness (if $\boldsymbol{P}$ is antisymmetric, $\bar{r}$ is zero).

We will call the above the static setting, since $\boldsymbol{P}$ is fixed. Replicator dynamics in zero sum games have already been analyzed, proving that it has limit cycles or is recurrent (e.g., see [1, 26-28, 33]).

1.2.2 Dynamic payoff matrix. We next define a dynamic setting, where at all times the payoff matrix is a convex combination of $n$ matrices:

$$
\boldsymbol{P}^{\boldsymbol{w}}=w_{1} \boldsymbol{P}_{1}+w_{2} \boldsymbol{P}_{2}+\cdots+w_{n} \boldsymbol{P}_{n},
$$

where

$$
\begin{gathered}
\boldsymbol{P}_{1}=\boldsymbol{P}+\left[\begin{array}{ccccc}
0 & \mu & \ldots & \mu & \mu \\
-\mu & 0 & \ldots & 0 & 0 \\
\vdots & \vdots & \vdots & \vdots & \vdots \\
-\mu & 0 & \ldots & 0 & 0
\end{array}\right], \boldsymbol{P}_{2}=\boldsymbol{P}+\left[\begin{array}{ccccc}
0 & -\mu & 0 & \ldots & 0 \\
\mu & 0 & \mu & \ldots & \mu \\
0 & -\mu & 0 & \ldots & 0 \\
\vdots & \vdots & \vdots & \vdots & \vdots \\
0 & -\mu & 0 & \ldots & 0
\end{array}\right], \ldots, \\
\boldsymbol{P}_{n}=\boldsymbol{P}+\left[\begin{array}{ccccc}
0 & 0 & \ldots & 0 & -\mu \\
0 & 0 & \ldots & 0 & -\mu \\
\vdots & \vdots & \vdots & \vdots & \vdots \\
0 & 0 & \ldots & 0 & -\mu \\
\mu & \mu & \ldots & \mu & 0
\end{array}\right] .
\end{gathered}
$$

Formally, $\boldsymbol{P}_{i}-\boldsymbol{P}$ is a matrix with entries $\mu$ at the $i$-th row and $-\mu$ at the $i$-th column (rest of the entries and diagonal entry $(i, i)$ are zero). It can be seen that for $\mu>0$ (for the rest of the paper $\mu \geq 0), \boldsymbol{P}_{i}$ favors type $i$ by increasing the payoff of $i$ when competing with other types. The weight vector $\boldsymbol{w}=\left(w_{1}, \ldots, w_{n}\right)$ changes according to the replicator dynamics under the following matrix

$$
=\left[\begin{array}{cccccc}
0 & x_{2}-x_{1} & x_{3}-x_{1} & \ldots & x_{n-1}-x_{1} & x_{n}-x_{1} \\
x_{1}-x_{2} & 0 & x_{3}-x_{2} & \ldots & x_{n-1}-x_{2} & x_{n}-x_{2} \\
\vdots & \vdots & \vdots & \vdots & \vdots & \vdots \\
x_{1}-x_{n} & x_{2}-x_{n} & x_{3}-x_{n} & \ldots & x_{n-1}-x_{n} & 0
\end{array}\right] .
$$

In words, $P^{w}$ changes with time. The idea behind the way $\boldsymbol{w}$ changes, is that species with small population should be favored. Since is also anti-symmetric and has 0 entries in the diagonal, the replicator dynamics update rule of $\boldsymbol{w}$ is

$$
w_{i}^{\prime}=w_{i} \sum_{j} w_{j}\left(x_{j}-x_{i}\right) \quad \forall i
$$


By model's definition,

$$
P^{w}=\left[\begin{array}{ccccc}
0 & -\alpha+\mu\left(w_{1}-w_{2}\right) & \mu\left(w_{1}-w_{3}\right) & \ldots & \alpha+\mu\left(w_{1}-w_{n}\right) \\
\alpha+\mu\left(w_{2}-w_{1}\right) & 0 & -\alpha+\mu\left(w_{2}-w_{3}\right) & \ldots & \mu\left(w_{2}-w_{n}\right) \\
\vdots & \vdots & \vdots & \vdots & \vdots \\
\mu\left(w_{n-1}-w_{1}\right) & \mu\left(w_{n-1}-w_{2}\right) & \mu\left(w_{n-1}-w_{3}\right) & \ldots & -\alpha+\mu\left(w_{n-1}-w_{n}\right) \\
-\alpha+\mu\left(w_{n}-w_{1}\right) & \mu\left(w_{n}-w_{2}\right) & \mu\left(w_{n}-w_{3}\right) & \ldots & 0
\end{array}\right]
$$

Note that $\boldsymbol{P}$ is anti-symmetric and has 0 entries in the diagonal. The replicator dynamics of $\boldsymbol{x}$ becomes

$$
x_{i}^{\prime}=x_{i} \cdot s_{i} \quad \forall i
$$

where $s=P^{w} x$ is the fitness vector of population $x$ under $\boldsymbol{P}^{\boldsymbol{w}}$. Summing up, the system of ordinary differential equations that we would like to analyze is captured by $\left(w_{i}\right.$ depends on $\left.x_{i}\right)$

$$
x_{i}^{\prime}=x_{i} \sum_{j} P_{i j}^{w} x_{j}, w_{i}^{\prime}=w_{i} \sum_{j} w_{j}\left(x_{j}-x_{i}\right) \quad \forall i
$$

\section{Connections to training GANs and min-max optimization in online settings:}

Generative Adversarial Networks (GANs) Goodfellow et al. [9] have been really successful over the last 3 years in Machine/Deep Learning, especially in generating images that look "superficially authentic". The framework is as follows: There are two deep neural networks, the Generator $(G)$ and the Discriminator (D). The generator gets noise as input and produces a sample in the same space of the sampled data set (e.g, an image), with the aim to approximate a sample from the underlying distribution. In the meantime, the discriminator is trying to discriminate a sample produced from generator and a true sample. This framework is modeled as a zero sum game between the two neural nets.

The aforementioned framework and also the framework of WGANs [2] has increased the interest of researchers in the areas of optimization and machine learning to work on min-max optimization (generator chooses $x$ and discriminator $y$ ):

$$
\min _{x} \max _{y} f(x, y)
$$

In [5] the authors showed that using gradient descent on $x$ and gradient ascent on $y$ might yield cycles for the problem (2). Moreover, in [22] it was essentially proved that any continuous time learning dynamics that consists an instance of FTRL (Followed the regularized leader), cycles when $f$ is essentially a bilinear function.

Our setting applies in the online framework of (2) where function $f$ changes with the time (iterations if the time is discrete, continuously otherwise). Our model is a specific case of such an online setting, where $f_{t}(x, y)=x^{T} A_{t} y, A_{t}$ is antisymmetric for all $t$ and in which we showed that cycles persist. It would be interesting to analyze more general online settings and design learning algorithms in which the last iterate converges (under assumptions on the sequence $\left\{A_{t}\right\}_{t \in \mathbb{N}}$ ).

Notation: We denote the probability simplex on a set of size $n$ as $\Delta_{n}$. Vectors in $\mathbb{R}^{n}$ are denoted in bold-face letters $x$ and are considered as column vectors. The $i$-th coordinate is denoted by $x_{i}$. To denote a row vector we use $x^{\top}$. The time derivative of a function $y=y(t)$ is denoted by $y^{\prime}$. 


\section{PRELIMINARIES}

\subsection{Dynamical Systems}

Let $f: \mathcal{S} \rightarrow \mathcal{S}$ be continuously differentiable with $\mathcal{S} \subset \mathbb{R}^{n}, \mathcal{S}$ an open set. A continuous (time) dynamical system is of the form

$$
y^{\prime} \equiv \frac{d y}{d t}=f(y)
$$

Since $f$ is continuously differentiable, the system of ordinary differential equations (ode (3)) along with the initial condition $y(0)=y_{0} \in \mathcal{S}$ has a unique solution for $t \in \mathcal{I}\left(y_{0}\right)$ (some time interval) and we can present it by $\phi\left(t, \boldsymbol{y}_{0}\right)$, called the flow of the system. $\phi_{t}\left(\boldsymbol{y}_{0}\right) \equiv \phi\left(t, \boldsymbol{y}_{0}\right)$ corresponds to a function of time which captures the trajectory/orbit of the system with $y_{0}$ the given starting point. It is continuously differentiable, its inverse exists (denoted by $\phi_{-t}\left(\boldsymbol{y}_{0}\right)$ ) and is also continuously differentiable (called diffeomorphism) in the so called maximal interval of existence $\mathcal{I}$. It is also true that $\phi_{t} \circ \phi_{s}=\phi_{t+s}$ for $t, s, t+s \in \mathcal{I} \cdot \boldsymbol{y}_{0} \in \mathcal{S}$ is called an equilibrium if $f\left(\boldsymbol{y}_{0}\right)=\mathbf{0}$. In that case holds $\phi_{t}\left(y_{0}\right)=y_{0}$ for all $t \in \mathcal{I}$, i.e., $y_{0}$ is a fixed point of the function $\phi_{t}(y)$ for all $t \in \mathcal{I}$.

If $f$ is globally Lipschitz then the flow is defined for all $t \in \mathbb{R}$, i.e., $\mathcal{I}=\mathbb{R}$. One way to enforce the dynamical system to have a well-defined flow for all $t \in \mathbb{R}$ is to renormalize the vector field by $\|f(y)\|+1$, i.e., the resulting dynamical system will be $\frac{d y}{d t}=\frac{f(y)}{\|f(y)\|+1}$, because the function becomes globally 1-Lipschitz. The two dynamical systems (before and after renormalization) are topologically equivalent ([25], p. 184). Formally this means that there exists a homeomorphism $H$ which maps trajectories of (3) onto trajectories of the renormalized flow and preserves the direction of time. In words it means that the two systems have the same behavior/geometry (same fixed points, convergence properties, phase portrait). For the rest of the paper, we may assume that the flow of dynamics (1) is well-defined for all $t \in \mathbb{R}$.

Definition 2.1 (Volume preserving). The differential equation (3) is said to be volume preserving on $\mathcal{S}$ if for any measurable set $B \subseteq \mathcal{S}$, we have $v\left(\phi_{t}(B)\right)=v(B)$ for all $t \in \mathbb{R}$, where $v$ is the Lebesgue measure and $\phi_{t}$ the flow of the ode.

The most common way to prove that the flow is volume preserving is via the Liouville's theorem.

TheOREM 2.2 (Liouville THEOREM [33]). Let $\boldsymbol{y}^{\prime}=f(\boldsymbol{y})$ be an ode with flow $\phi_{t}$. It holds that

$$
\frac{d v\left(\phi_{t}(B)\right)}{d t}=\int_{\phi_{t}(B)}(\nabla \cdot f) d v, \text { for each Lebesgue measurable set } B .
$$

Therefore, as long as $\nabla \cdot f=0$, the flow preserves volume.

In 1890, Poincaré [29] showed that whenever a dynamical system preserves volume, almost all trajectories return arbitrarily close to their initial position an infinite number of times.

Theorem 2.3 (Poincaré Recurrence [3,29]). If a flow preserves volume and has only bounded orbits then for each open set there exist orbits that intersect the set infinitely often.

For more information on dynamical systems see [25] and for readers familiar with game theory see $[13,33]$.

\subsection{Replicator Dynamics and Evolution}

Replicator equations, first were introduced by Fisher [8] in 30's for genotype evolution. The simplest form of replicator equations is the following:

$$
x_{i}^{\prime}(t)=x_{i}(t)\left((A x(t))_{i}-x(t)^{\top} A x(t)\right) .
$$


where $A$ is a payoff matrix (generally non-negative), $x$ a vector that lies in simplex and $(A x)_{i}$ denotes $\sum_{j} A_{i j} x_{j}$. Observe that in the nonlinear dynamics above, simplex is invariant (if we start from a probability distribution, the vector remains a probability distribution). This dynamics is called replicator dynamics and has been used numerous times in biology, evolution, game theory and genetic algorithms. The dynamics we analyze in this paper is a version of replicator dynamics (on generalized RPS with dynamics payoff matrix).

\section{OUR RESULTS}

In this section we state and prove our main result. Our main theorem can be stated formally as follows (see also Figures 1,2):

THEOREM 3.1. For all but measure zero of initial positions, the trajectories of the dynamics (1) return arbitrarily close to their initial position an infinite number of times.

Definition 3.2 (Natural transformation [13]). We define the natural transformation $\Pi: \operatorname{int}\left(\Delta_{n}\right) \rightarrow$ $\mathbb{R}^{n-1}$ to be $\Pi(y)=\left(\log \left(\frac{y_{1}}{y_{n}}\right), \ldots, \log \left(\frac{y_{n-1}}{y_{n}}\right)\right)$. In words, we map every point $y$ of the interior of simplex to a point in $\mathbb{R}^{n-1}$. It is not hard to see that the map is injective and surjective. The reason is that $\Pi^{-1}$ exists and is equal to $\Pi^{-1}(z)=\left(\frac{e^{z_{1}}}{1+\sum_{j=1}^{n-1} e^{z_{j}}}, \ldots, \frac{e^{z_{n-1}}}{1+\sum_{j=1}^{n-1} e^{z_{j}}}, \frac{1}{1+\sum_{j=1}^{n-1} e^{z_{j}}}\right)$. The points on the boundary of simplex correspond to vectors with infinity Euclidean norm in $\mathbb{R}^{n-1}$.

Lemma 3.3. Let $\left(x^{0}, w^{0}\right)$ be an initial point in the interior of $\Delta_{n} \times \Delta_{n}$. The dynamics mapped to $\mathbb{R}^{n-1} \times \mathbb{R}^{n-1}$ (under natural mapping $(\Pi, \Pi)$ ) has bounded orbits $\left(\Pi\left(x^{t}\right), \Pi\left(\boldsymbol{w}^{t}\right)\right)$.

Proof. We define the log-barrier function

$$
D(x)=\sum_{i=1}^{n} \log \left(\frac{1}{x_{i}}\right) .
$$

Computing the derivative we get,

$$
\begin{aligned}
D^{\prime}(x) & =-\sum_{i} \frac{x_{i}^{\prime}}{x_{i}}=-\sum_{i} s_{i}=-\sum_{i} \sum_{j} P_{i j}^{w} x_{j} \\
& =-\left(\sum_{j} x_{j}\left(\mu \sum_{i}\left(w_{i}-w_{j}\right)+\alpha-\alpha\right)\right) \\
& =-\mu\left(\sum_{j} x_{j}\left(1-n w_{j}\right)\right) \\
& =-\mu\left(1-n \sum_{j} w_{j} x_{j}\right) .
\end{aligned}
$$

We also define the log-barrier function

$$
D(\boldsymbol{w})=\sum_{i=1}^{n} \log \left(\frac{1}{w_{i}}\right),
$$


and we have

$$
\begin{aligned}
D^{\prime}(\boldsymbol{w}) & =-\sum_{i} \frac{w_{i}^{\prime}}{w_{i}}=-\sum_{i} \sum_{j} w_{j}\left(x_{j}-x_{i}\right) \\
& =-\sum_{j} \sum_{i} w_{j}\left(x_{j}-x_{i}\right) \\
& =-\sum_{j} w_{j}\left(n x_{j}-1\right) \\
& =-\left(n \sum_{j} w_{j} x_{j}-1\right) .
\end{aligned}
$$

Therefore,

$$
\begin{aligned}
D^{\prime}(x)+\mu D^{\prime}(\boldsymbol{w}) & =-\mu\left(1-n \sum_{j} w_{j} x_{j}\right)-\mu\left(n \sum_{j} w_{j} x_{j}-1\right) \\
& =\left(n \sum_{j} w_{j} x_{j}-1\right)(\mu-\mu)=0 .
\end{aligned}
$$

Hence,

$$
D(x)+\mu D(w)
$$

is a constant motion of time (independent of time). It is also clear that $D(x), D(\boldsymbol{w}) \geq 0$ and become infinity only on the boundary of $\Delta_{n} \times \Delta_{n}$. Since $D\left(x^{0}\right)+\mu D\left(\boldsymbol{w}^{0}\right)$ is bounded (i.e., $\left(\boldsymbol{x}^{0}, \boldsymbol{w}^{0}\right)$ is an interior point), we get that $D\left(x^{t}\right)+\mu D\left(\boldsymbol{w}^{t}\right)$ is bounded for all times $t$, hence there is no subsequence of times $t_{k}$ so that $\left(x^{t_{k}}, \boldsymbol{w}^{t_{k}}\right)$ converges to the boundary of $\Delta_{n} \times \Delta_{n}$. Therefore there is no subsequence of times $t_{k}$ so that $\left\|\left(\Pi\left(x^{t_{k}}\right), \Pi\left(\boldsymbol{w}^{t_{k}}\right)\right)\right\|_{2}$ goes to infinity, i.e., the dynamics on $\mathbb{R}^{n-1} \times \mathbb{R}^{n-1}$ (under natural mapping $(\Pi, \Pi))$ has bounded orbits.

Proof of Theorem 3.1. Under the mapping $\Pi$ for $\boldsymbol{x}$, $\boldsymbol{w}$, set $\boldsymbol{y} \equiv \Pi(\boldsymbol{x})$ and $z \equiv \Pi(\boldsymbol{w})$. We shall show that the dynamics $\left(\boldsymbol{y}^{\prime}, \boldsymbol{z}^{\prime}\right)=g(\boldsymbol{y}, \boldsymbol{z})$ satisfies the conditions of the Poincaré recurrence theorem (Theorem 2.3), where $g$ is the vector field of the resulting dynamics, after the transformation (П, $)$.

The vector field becomes $y_{i}^{\prime}=\frac{x_{n}}{x_{i}} \frac{x_{i}^{\prime} x_{n}-x_{i} x_{n}^{\prime}}{x_{n}^{2}}=\frac{x_{i}^{\prime}}{x_{i}}-\frac{x_{n}^{\prime}}{x_{n}}=\sum_{j}\left(P_{i j}^{w}-P_{n j}^{w}\right) x_{j}=\alpha\left(x_{1}+x_{i-1}-x_{n-1}-\right.$ $\left.x_{i+1}\right)+\mu\left(w_{i}-w_{n}\right)\left(\right.$ with the convention that $\left.x_{0}=x_{n}\right)$. Similarly $z_{i}^{\prime}=\frac{w_{n}}{w_{i}} \frac{w_{i}^{\prime} w_{n}-w_{i} w_{n}^{\prime}}{w_{n}^{2}}=\frac{w_{i}^{\prime}}{w_{i}}-\frac{w_{n}^{\prime}}{w_{n}}=$ $\sum_{j} w_{j}\left(x_{n}-x_{i}\right)=x_{n}-x_{i}$

After substitution (using $\Pi^{-1}$ ) we get

$$
y_{i}^{\prime}=\alpha \cdot \frac{e^{y_{1}}+e^{y_{i-1}}-e^{y_{n-1}}-e^{y_{i+1}}}{1+\sum_{j=1}^{n-1} e^{y_{j}}}+\mu \frac{e^{z_{i}}-1}{1+\sum_{j=1}^{n-1} e^{z_{j}}}, z_{i}^{\prime}=\frac{1-e^{y_{i}}}{1+\sum_{j=1}^{n-1} e^{y_{j}}} .
$$

We shall prove that the flow of the dynamics (4) preserves the volume, by showing that $\nabla \cdot g=0$ (then it follows from Liouville's theorem 2.2).

Set $S=\sum_{j=1}^{n-1} e^{y_{j}}$. We compute the partial derivatives and we get that 


$$
\begin{array}{ll}
\frac{\partial g}{\partial y_{1}} & =\alpha \cdot \frac{e^{y_{1}}(1+S)-e^{y_{1}}\left(1+e^{y_{1}}-e^{y_{2}}-e^{y_{n-1}}\right)}{(1+S)^{2}}, \\
\frac{\partial g}{\partial y_{j}} & =-\alpha \cdot \frac{e^{y_{j}}\left(e^{y_{1}}+e^{y_{j-1}}-e^{y_{j+1}}-e^{y_{n-1}}\right)}{(1+S)^{2}}, \text { for } 2 \leq j \leq n-2, \\
\frac{\partial g}{\partial y_{n-1}} & =\alpha \cdot \frac{-e^{y_{n-1}(1+S)-e^{y_{n-1}}\left(e^{y_{1}}+e^{y_{n-2}}-1-e^{y_{n-1}}\right)}}{(1+S)^{2}}, \\
\frac{\partial g}{\partial z_{j}} & =0 \text { for } 1 \leq j \leq n-1 .
\end{array}
$$

Therefore we get that

$$
\begin{aligned}
\nabla \cdot g & =\sum_{j=1}^{n-1} \frac{\partial g}{\partial y_{j}}+\sum_{j=1}^{n-1} \frac{\partial g}{\partial z_{j}} \\
& =\frac{-\alpha \cdot \sum_{j=1}^{n-1} e^{y_{j}}\left(e^{y_{1}}+e^{y_{j-1}}-e^{y_{j+1}}-e^{y_{n-1}}\right)+\alpha \cdot\left(e^{y_{1}}-e^{y_{n-1}}\right)\left(1+\sum_{j=1}^{n-1} e^{y_{j}}\right)}{(1+S)^{2}},
\end{aligned}
$$

with the convention that $y_{0}=y_{n}=0$. We get that all the terms of the form $e^{y_{j}} e^{y_{j+1}}$ cancel out (telescopically) and also terms $e^{y_{1}} e^{y_{j}}, e^{y_{n-1}} e^{y_{j}}$ also cancel out for all $j$, i.e., it turns out that $\nabla \cdot g=0$. Hence we conclude from Liouville theorem that the dynamical system (4) preserves volume.

Using the fact that the flow preserves the volume and that the orbits are bounded (Lemma 3.3), we apply Poincaré recurrence theorem on $(\tilde{y}, \tilde{z})=g(y, z)$ for a small open ball around any initial point $\left(\tilde{y}_{0}, \tilde{z}_{0}\right)$ and the claim follows for the transformed dynamics. Since $\Pi^{-1}$ is continuous, if the distance between two points goes to zero in $\mathbb{R}^{n-1} \times \mathbb{R}^{n-1}$, so it does in $\Delta_{n} \times \Delta_{n}$ and the claim follows for the dynamics (1).

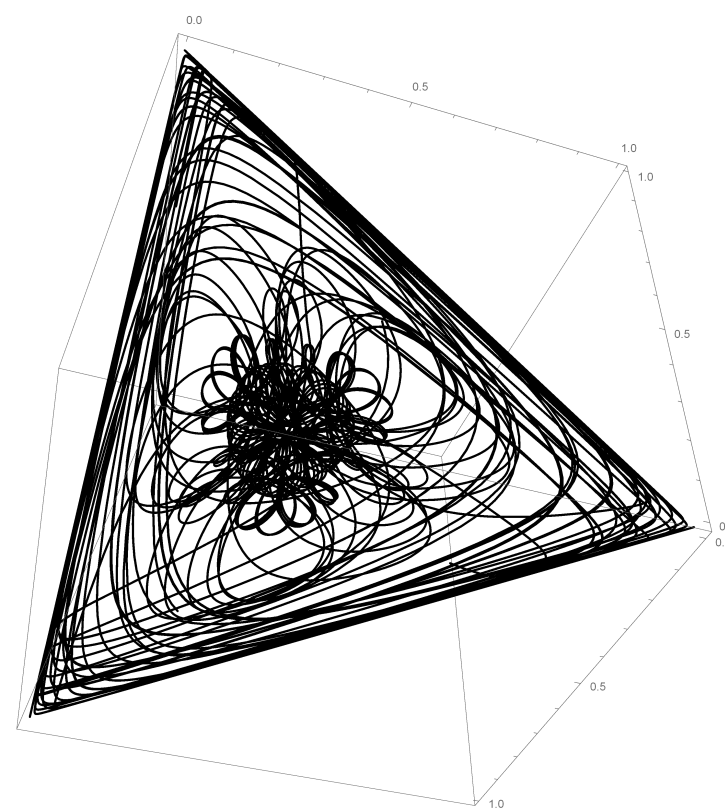

Fig. 1. Trajectories of the vector $x$ for different initial positions with $\mu=0.1$. Trajectories intersect due to the fact 6 dimensions are projected to a 3D figure. The "cycling" behavior is observed. 


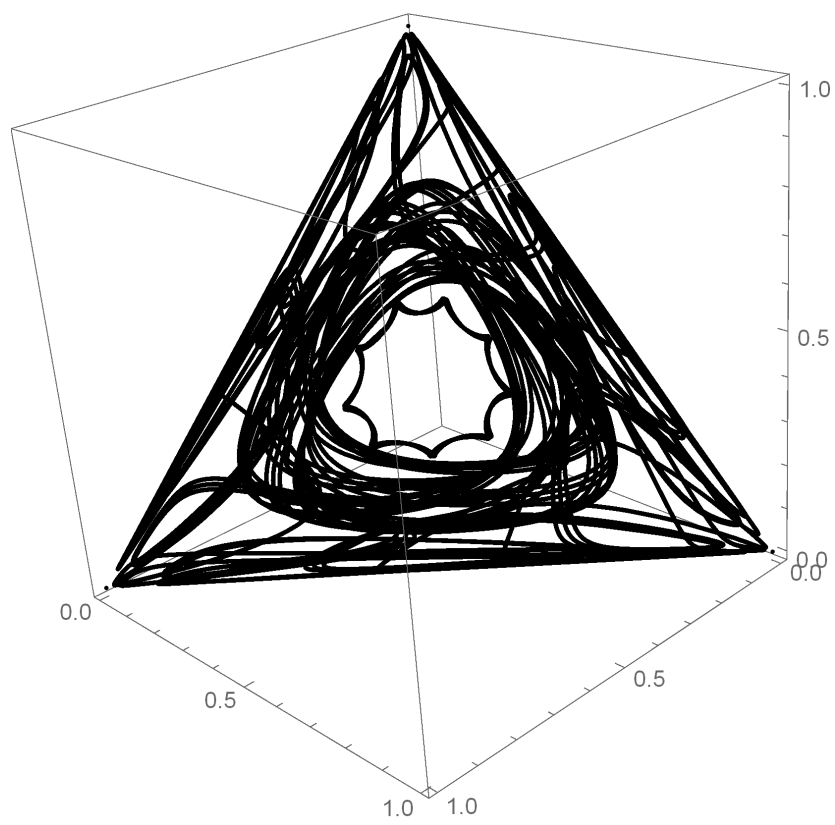

Fig. 2. Trajectories of the vector $w$ for different initial positions with $\mu=0.1$. Trajectories intersect due to the fact 6 dimensions are projected to a 3D figure. The "cycling" behavior is observed.

\section{DISCUSSION}

We believe that our work and that of [38] are opening up the possibility of modeling more complex biological phenomena using the setting of differential games, thereby potentially leading to new insights into biological phenomena. Additionally, we believe that the kinds of techniques used in this paper, and highlighted in Section 1.1, will be useful for studying other models of non-transitive dynamics.

We are not aware of other uses of the Poincaré theorem in differential games. It will be nice to see other applications of this powerful theorem to learning dynamics, other than replicator dynamics (such as FTRL), where the state space (payoff matrix) changes as per a differential equation.

There are several generalizations of our model which are worth studying. For example, what happens if each matrix $\boldsymbol{P}_{i}$ has its own parameter $\mu_{i}$ ? So far we have not been able to prove cycling for this general case but simulations indicate that the system does cycle. Understanding the range of parameters which lead to cycling and those that do not will be interesting. 


\section{REFERENCES}

[1] E. Akin and V. Losert. 1984. Evolutionary dynamics of zero-sum games. J. of Math. Biology (1984).

[2] Martín Arjovsky, Soumith Chintala, and Léon Bottou. 2017. Wasserstein Generative Adversarial Networks. In Proceedings of the 34th International Conference on Machine Learning, ICML 2017, Sydney, NSW, Australia, 6-11 August 2017. 214-223.

[3] L. Barreira. 2006. Poincaré recurrence: old and new. XIVth International Congress on Mathematical Physics. World Scientific. (2006), 415-422.

[4] D. D. Cameron, A. White, and J. Antonovics. 2009. ParasiteâĂŞgrassâĂŞforb interactions and rockâĂŞpaperâĂŞscissor dynamics: predicting the effects of the parasitic plant Rhinanthus minor on host plant communities. fournal of Ecology (2009), 1311-1319.

[5] Constantinos Daskalakis, Andrew Ilyas, Vasilis Syrgkanis, and Haoyang Zeng. 2018. Training GANs with Optimism. To appear in ICLR (2018). arXiv:1711.00141

[6] Engelbert Dockner. 2000. Differential games in economics and management science. Cambridge University Press.

[7] Deborah L. Finke and William E. Snyder. 2008. Niche Partitioning Increases Resource Exploitation by Diverse Communities. Science 321, 5895 (2008), 1488-1490.

[8] R.A. Fisher. 1999. The Genetical Theory of Natural Selection. A complete variorum edition. Clarendon Press, Oxford.

[9] Ian J. Goodfellow, Jean Pouget-Abadie, Mehdi Mirza, Bing Xu, David Warde-Farley, Sherjil Ozair, Aaron C. Courville, and Yoshua Bengio. 2014. Generative Adversarial Nets. In Advances in Neural Information Processing Systems 27: Annual Conference on Neural Information Processing Systems 2014, December 8-13 2014, Montreal, Quebec, Canada. 2672-2680.

[10] Jeremy JD Greenwood. 1984. The functional basis of frequency-dependent food selection. Biological fournal of the Linnean Society 23, 2-3 (1984), 177-199.

[11] Mart R Gross. 1982. Sneakers, satellites and parentals: polymorphic mating strategies in North American sunfishes. Ethology 60, 1 (1982), 1-26.

[12] James Haven, Levy C Vargas, Emmanuel F Mongodin, Vincent Xue, Yozen Hernandez, Pedro Pagan, Claire M FraserLiggett, Steven E Schutzer, Benjamin J Luft, Sherwood R Casjens, and others. 2011. Pervasive recombination and sympatric genome diversification driven by frequency-dependent selection in Borrelia burgdorferi, the Lyme disease bacterium. Genetics 189, 3 (2011), 951-966.

[13] J. Hofbauer and K. Sigmund. 1998. Evolutionary Games and Population Dynamics. Cambridge University Press, Cambridge.

[14] R. Isaacs. 1999. Differential Games. Dover.

[15] Rufus Isaacs. 1999. Differential games: a mathematical theory with applications to warfare and pursuit, control and optimization. Courier Corporation.

[16] B. Kerr, C. Neuhauser, B. J. M. Bohannan, and A. M. Dean. 2006. Local migration promotes competitive restraint in a host-pathogen'tragedy of the commons'. Nature (2006).

[17] B. Kerr, M. A. Riley, M. W. Feldman, and B. J. M. Bohannan. 2002. Local dispersal promotes biodiversity in a real-life game of rock-paper-scissors. Nature (2002).

[18] B. C. Kirkup and M. A. Riley. 2004. Antibiotic-mediated antagonism leads to a bacterial game of rock-paper-scissor in vivo. Nature (2004).

[19] Britt Koskella and Curtis M Lively. 2009. Evidence for negative frequency-dependent selection during experimental coevolution of a freshwater snail and a sterilizing trematode. Evolution 63, 9 (2009), 2213-2221.

[20] R. A. Lankau and S. Y. Strauss. 2007. Mutual feedbacks maintain both genetic and species diversity in a plant community. Science (2007), 1561-1563.

[21] R. Mehta, I. Panageas, G. Piliouras, P. Tetali, and V. V. Vazirani. 2017. Mutation, Sexual Reproduction and Survival in Dynamic Environments. Innovations in Theoretical Computer Science (ITCS) (2017).

[22] Panayotis Mertikopoulos, Christos Papadimitriou, and Georgios Piliouras. 2018. Cycles in Adversarial Regularized Learning. In Proceedings of the Twenty-Ninth Annual ACM-SIAM Symposium on Discrete Algorithms, SODA 2018, New Orleans, LA, USA, January 7-10, 2018. 2703-2717.

[23] Levi T Morran, Olivia G Schmidt, Ian A Gelarden, Raymond C Parrish, and Curtis M Lively. 2011. Running with the Red Queen: host-parasite coevolution selects for biparental sex. Science 333, 6039 (2011), 216-218.

[24] Robert Olendorf, F Helen Rodd, David Punzalan, Anne E Houde, Carla Hurt, David N Reznick, and Kimberly A Hughes. 2006. Frequency-dependent survival in natural guppy populations. Nature 441, 7093 (2006), 633-636.

[25] L. Perko. 1991. Differential Equations and Dynamical Systems (3nd. ed.). Springer.

[26] G. Piliouras, C. Nieto-Granda, H. I. Christensen, and J. S. Shamma. 2014. Persistent patterns: multi-agent learning beyond equilibrium and utility. In International conference on Autonomous Agents and Multi-Agent Systems, AAMAS. 181-188.

[27] Georgios Piliouras and Leonard J. Schulman. 2018. Learning Dynamics and the Co-Evolution of Competing Sexual Species. In 9th Innovations in Theoretical Computer Science Conference, ITCS 2018, fanuary 11-14, 2018, Cambridge, MA, 
USA. 59:1-59:3.

[28] G. Piliouras and J. S. Shamma. 2014. Optimization Despite Chaos: Convex Relaxations to Complex Limit Sets via Poincaré Recurrence. ACM-SIAM symposium on Discrete algorithms (SODA) (2014).

[29] H. Poincaré. 1890. Sur le probléme des trois corps et les équations de la dynamique. Acta Math 13 (1890), 1-270.

[30] Paul B Rainey, Angus Buckling, Rees Kassen, and Michael Travisano. 2000. The emergence and maintenance of diversity: insights from experimental bacterial populations. Trends in ecology \& evolution 15, 6 (2000), 243-247.

[31] T. Reichenbach, M. Mobilia, and E. Frey. 2007. Local migration promotes competitive restraint in a host-pathogen' tragedy of the commons'. Nature (2007), 1046-1049.

[32] Volker HW Rudolf and Janis Antonovics. 2005. Species coexistence and pathogens with frequency-dependent transmission. The American Naturalist 166, 1 (2005), 112-118.

[33] W. H. Sandholm. 2010. Population Games and Evolutionary Dynamics. MIT Press.

[34] L. S. Shapley. 1953. Stochastic games. Proceedings of the National Academy of Sciences (PNAS) (1953), 1095-1100.

[35] B. Sinervo and C. M. Lively. 1996. The rock-paper-scissors game and the evolution of alternative male strategies. Nature (1996).

[36] Peter H Thrall, Arjen Biere, and Marcy K Uyenoyama. 1995. Frequency-dependent disease transmission and the dynamics of the Silene-Ustilago host-pathogen system. The American Naturalist 145, 1 (1995), 43-62.

[37] David Tilman. 1982. Resource competition and community structure. Princeton university press.

[38] J. S. Weitz, C. Eksin, K. Paarporn, S. P. Brown, and W. C. Ratcliff. 2016. An oscillating tragedy of the commons in replicator dynamics with game-environment feedback. Proceedings of the National Academy of Sciences. 113 (2016), 7518-7525.

[39] RE Wong. 1967. Some aerospace differential games. fournal of Spacecraft and Rockets 4, 11 (1967). 